\title{
Presentation, diagnostic assessment and surgical outcomes in primary hyperparathyroidism: a single centre's experience
}

\author{
Laura J Reid', Bala Muthukrishnan',*, Dilip Patel2, Mike S Crane ${ }^{3}$, Murat Akyol4, Andrew Thomson ${ }^{5}$ \\ Jonathan R Seck|1,6 and Fraser W Gibb
}

${ }^{1}$ Edinburgh Centre for Endocrinology and Diabetes, Royal Infirmary of Edinburgh, Edinburgh, UK

${ }^{2}$ Department of Radiology, Royal Infirmary of Edinburgh, Edinburgh, UK

${ }^{3}$ Department of Clinical Biochemistry, Royal Infirmary of Edinburgh, Edinburgh, UK

${ }^{4}$ Department of Surgery, Royal Infirmary of Edinburgh, Edinburgh, UK

${ }^{5}$ Department of Pathology, Royal Infirmary of Edinburgh, Edinburgh, UK

${ }^{6}$ Centre for Cardiovascular Science, Queen's Medical Research Unit, University of Edinburgh, Edinburgh, UK

Correspondence should be addressed to F W Gibb: fraser.gibb@ed.ac.uk

*(B Muthukrishnan is now at Borders General Hospital, Melrose, UK)

\begin{abstract}
Objective: Primary hyperparathyroidism (PHPT) is a common reason for referral to endocrinology but the evidence base guiding assessment is limited. We evaluated the clinical presentation, assessment and subsequent management in PHPT.

Design: Retrospective cohort study.

Patients: PHPT assessed between 2006 and $2014(n=611)$ in a university hospital.

Measurements: Symptoms, clinical features, biochemistry, neck radiology and surgical outcomes.

Results: Fatigue (23.8\%), polyuria (15.6\%) and polydipsia (14.9\%) were associated with PHPT biochemistry. Bone fracture was present in $16.4 \%$ but was not associated with biochemistry. A history of nephrolithiasis (10.0\%) was associated only with younger age $(P=0.006)$ and male gender $(P=0.037)$. Thiazide diuretic discontinuation was not associated with any subsequent change in calcium $(P=0.514)$. Urine calcium creatinine clearance ratio (CCCR) was $<0.01$ in $18.2 \%$ of patients with confirmed PHPT. Older age $(P<0.001)$ and lower PTH $(P=0.043)$ were associated with failure to locate an adenoma on ultrasound (44.0\% of scans). When an adenoma was identified on ultrasound the lateralisation was correct in $94.5 \%$. Non-curative surgery occurred in $8.2 \%$ and was greater in those requiring more than one neck imaging modality (OR 2.42, $P=0.035$ ). Conclusions: Clinical features associated with PHPT are not strongly related to biochemistry. Thiazide cessation does not appear to attenuate hypercalcaemia. PHPT remains the likeliest diagnosis in the presence of low CCCR. Ultrasound is highly discriminant when an adenoma is identified but surgical failure is more likely when more than one imaging modality is required.
\end{abstract}

$\begin{aligned} & \text { Key Words } \\ & \text { - hyperparathyroidism } \\ & \text { - primary } \\ & \text { - calcium } \\ & \text { - hypercalcaemia } \\ & \text { - thiazides } \\ & \text { - vitamin D } \\ & \text { - parathyroid hormone }\end{aligned}$

Endocrine Connections (2018) 7, 1105-1115

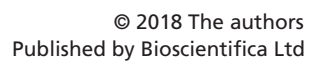

Published by Bioscientifica Ltd

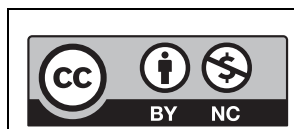

This work is licensed under a Creative Commons Attribution-NonCommercial 4.0 International License. 


\section{Introduction}

Primary hyperparathyroidism (PHPT) is a common endocrine condition with a prevalence of $0.1-1 \%$, a female preponderance and a strong association with ageing $(1,2)$. In the modern era, PHPT is often detected incidentally through routine biochemical testing and is, in this milder form, often described as asymptomatic, in distinction from more severe PHPT associated with classical bone, renal and neuropsychiatric manifestations. Many patients with relatively modest hypercalcaemia will report symptoms that are potentially attributable to PHPT. However, the degree to which these correlate with biochemical markers of severity and their reversibility with surgical cure remains unclear (3). The majority of PHPT may remain undiagnosed as suggested by a recent study which identified measurement of parathyroid hormone (PTH) in only $31 \%$ of hypercalcaemic patients (4). In recent years, the normocalcaemic variant of PHPT has gained increasing recognition, although this is beyond the scope of our investigation (5).

Guidance on the assessment and management of PHPT comes from the 4 th International Workshop on PHPT. This emphasises the importance of securing the diagnosis of PHPT before considering whether surgical intervention is appropriate (a decision largely based on age, serum calcium and presence of bone and/or renal complications) (6). Only when a decision to pursue surgical cure is made should neck imaging be performed. However, the optimal imaging modality in this context remains unclear and in a small but important minority of cases, the source of PTH excess remains elusive despite multiple imaging. The international workshop recognises the value of neck imaging by ultrasound, ${ }^{99 \mathrm{~m} T c-s e s t a m i b i}$ and CT in preoperative localisation (7), but does not specify an optimal approach.

Familial hypocalciuric hypercalcaemia $(\mathrm{FHH})$ is an important differential diagnosis to exclude in patients with apparent PHPT as it is often associated with mild hypercalcaemia and PTH concentrations that overlap with the lower end of those observed in PHPT. The initial recommended screening test to exclude $\mathrm{FHH}$, in addition to a full family history, is the urine calcium creatinine clearance ratio (CCCR), which should be $>0.01$ in patients with PHPT (8). Another potential contributor to hypercalcaemia in patients with probable PHPT is thiazide diuretic use, although recent reports suggest the impact to be minimal and paradoxically thiazides may even reduce blood PTH concentrations (9).

We have collated one of the largest cohorts of patients presenting for specialist endocrine assessment of PHPT, characterised in detail with respect to demographics, clinical features, biochemistry, imaging, surgery, pathology and subsequent outcomes. We sought to utilise this resource to address the relationship between presenting features and biochemical indices of PHPT; assess the discriminatory value of CCCR; determine the frequency of delayed diagnosis of PHPT; determine factors associated with the decision to pursue surgery and assess the effectiveness of pre-surgical neck imaging in a 'real world' context and relate these findings to surgical outcomes.

\section{Materials and methods}

\section{Patients}

All patients presenting with a new diagnosis of PHPT ( $n=611)$ to Edinburgh Centre for Endocrinology \& Diabetes Clinics (Royal Infirmary of Edinburgh \& Western General Hospital) between 2006 and 2014 were identified from our comprehensive clinic database. Patients presenting following family screening for genetic causes of PHPT were excluded from this cohort but all index cases were included. Basic demographic details were obtained from electronic patient records. Social deprivation status was assessed using the 'Scottish Index of Multiple Deprivation' (SIMD 2016), which assigns individuals a rank from 1 (most deprived) to 6976 (least deprived) based on postcode (10). Symptoms at presentation and other clinical features were gleaned from letters pertaining to PHPT in the electronic patient record. Ethical approval for this project was not required as it involved only retrospective review of our own patient cohort.

\section{Biochemistry}

Calcium (plasma and urine), phosphate, magnesium, alkaline phosphatase and creatinine were measured by standard laboratory methods. Adjusted calcium was derived using the following calculation: $($ calcium $)+((40-($ albumin $)) \times 0.02)$. Peak calcium was defined as the highest ever recorded calcium (from data available 1999 to 2016). CCCR was obtained either from spot urine sample or 24-h urine collection, in association with contemporaneous plasma samples, using the following calculation: ((urine calcium $(\mathrm{mM})) \times(($ serum creatinine $(\mu \mathrm{M})) \times 1000)) /(($ serum calcium $(\mathrm{mM})) \times($ urine creatinine $(\mathrm{mM})))$. Patients were not on thiazide diuretics or calcium supplementation prior to urine collection. Three PTH assays were used across the 9 years of patient

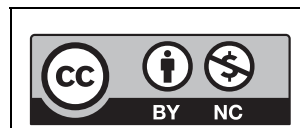

This work is licensed under a Creative Commons Attribution-NonCommercial 4.0 International License. 
acquisition, all of which measured 'intact' 1-84 PTH: the Abbott Architect i2000 chemiluminescent microparticleenhanced immunoassay (50.6\%), the Siemens Immulite 2000 solid-phase 2-site chemiluminescent enzyme linked immunometric assay (36.0\%) and the Roche Cobas E411 electrochemiluminescent assay (13.4\%). All reported PTH values were normalised to the Abbott Architect i2000 assay using regression equations (from locally derived comparison data). Vitamin D was measured using LC-MS/MS; deficiency was defined as $<25 \mathrm{nM}$ and insufficiency as 25-50 nM. Biochemical results dating back to 1999 were available on our system and were interrogated to identify the interval between a first hypercalcaemic result and the first assessment of PTH, as a proxy of delayed diagnosis.

\section{Imaging}

The standard sequence of imaging in our centre was initial high-resolution ultrasound with a minimum transducer frequency of $12.5 \mathrm{MHz} .{ }^{99 \mathrm{~m}} \mathrm{Tc}$-sestamibi (Planar and SPECT/CT) was performed only where ultrasound failed to identify a source for autonomous PTH secretion or where ultrasound findings were equivocal. Latterly, four-dimensional CT (4DCT) was employed where ultrasound and ${ }^{99 m}$ Tc-sestamibi-SPECT CT failed to identify an adenoma. Results are presented separately for the ${ }^{99 \mathrm{~m}} \mathrm{Tc}$-sestamibi uptake component (either planar ${ }^{99 m}$ Tc-sestamibi or ${ }^{99 m}$ Tc-sestamibi SPECT) and the CT component of concomitant ${ }^{99 \mathrm{~m}} \mathrm{Tc}-$ sestamibi SPECT CT imaging. Planar ${ }^{99 \mathrm{~m}} \mathrm{Tc}-$-sestamibi and ${ }^{99 \mathrm{~m}} \mathrm{Tc}-\mathrm{sestamibi}$ SPECT were considered as a single entity for data analysis. In cases with surgically confirmed adenoma/hyperplasia location data, the accuracy of imaging modalities were assessed in terms of ability to correctly lateralise disease and also to identify the precise location (i.e. right/left, superior/inferior or ectopic).

\section{Pathology}

Information on the location of the diseased parathyroid gland(s), dimensions, weight and ultimate histological subtype (adenoma, atypical adenoma, hyperplasia or carcinoma) was obtained from pathology reports. Tumour volume was calculated using the mathematical formula for the volume of an ellipsoid shape: $4 / 3 \times \pi \times a \times b \times c$ (where $a, b$ and $c$ are the radii of the three axes).

\section{Statistics}

Continuous variables were not normally distributed (assessed by Shapiro-Wilk test) and are reported as median (inter-quartile range). Comparisons between groups were analysed by Mann-Whitney $U$ test or Kruskal-Wallis test (multiple groups). Categorical data were compared by chi-square test. Correlations were analysed by Spearman rank test. Logistic regression analysis was employed to determine independent predictors of the presence of clinical features, recourse to neck imaging, failure of neck imaging and recourse to surgery. Statistical significance was accepted at $P<0.05$. All statistical analyses were performed on RStudio (version 1.1.338).

\section{Results}

\section{Characteristics of cohort}

The median age at diagnosis of PHPT was 68 years (IQR 58-77). The majority of patients $(82.3 \%, n=503)$ were female. Full baseline characteristics are presented in Table 1.

$22.7 \% \quad(n=139)$ presented with adjusted calcium greater than $0.25 \mathrm{mM}$ above the upper limit of normal (a level above which guidelines recommend surgery). $29.3 \%(171 / 584)$ had a phosphate below the reference range at presentation.

$11.1 \%(n=68)$ had a PTH within the reference range, $54.5 \%(n=333)$ had PTH above normal but less than twice the upper limit of normal, and $34.4 \%(n=210)$ had a PTH greater than twice the upper limit of normal. Only 1 of $251(0.4 \%)$ surgically cured patients had a $\mathrm{PTH}<5 \mathrm{pM}$ at initial diagnosis (and only 1.8\% of the total cohort). Vitamin D was available at baseline in 413 patients and was sufficient in $21.3 \%(n=88)$, insufficient in $46.0 \%$ $(n=190)$ and deficient in $32.7 \% \quad(n=135) .18 .5 \%$ of patients had chronic kidney disease stage three, $0.5 \%$ had CKD4 and the remainder had normal renal function, as determined by eGFR.

Genetic testing was performed in 51 patients: MEN-1 (31 normal, 1 pathogenic mutation, 2 variants of uncertain significance); MEN-2 (22 normal, 0 pathogenic mutations); CASR (10 normal, 1 pathogenic mutation) and CDC73 (12 normal, 1 pathogenic mutation).

\section{Presenting symptoms}

The most commonly reported symptoms at initial presentation were bone or joint pain, fatigue, polyuria, polydipsia and constipation or abdominal pain (Table 1). Low mood, memory impairment and weakness were less commonly recorded at diagnosis. 28.9\% (176/610) 
Table 1 Summary of presenting features in patients with PHPT.

Age at first high calcium (years)
Age at first PTH result (years)
Interval from high calcium to PTH measurement (months)
Weight $(\mathrm{kg}$ )
BMI ( $\mathrm{kg} / \mathrm{m}^{2}$ )
SIMD rank (out of 6976$)$
Gender
Fatigue at presentation
Weakness at presentation
Memory impairment at presentation
Depression at presentation
Polydipsia at presentation
Polyuria at presentation
Bone/joint pain at presentation
Previous fracture at presentation
Constipation/abdominal pain at presentation
Thiazide diuretic at presentation
Previous/current lithium therapy
Renal stones at or prior to diagnosis
Cardiovascular disease at diagnosis
Calcium (mM) at diagnosis
Adjusted calcium (mM) at diagnosis
Peak calcium (mM)
PTH at diagnosis (pM)
Vitamin D (nM)
Phosphate at diagnosis (mM)
Magnesium (mM)
Creatinine ( $\mu \mathrm{M}$ )
Alkaline phosphatase (U/L)
Urine calcium (mM)
Urine calcium (mmol/24h)
Urine CCCR

\begin{tabular}{|c|c|c|}
\hline $\boldsymbol{N}$ & Reference range & Median (IQR) \\
\hline 611 & & $66.3(56.4-64.9)$ \\
\hline 611 & & $68.0(58.0-77.0)$ \\
\hline 611 & & $1(0-18.5)$ \\
\hline 448 & & $73.3(62.0-74.6)$ \\
\hline 445 & & $27.8(24.2-28.4)$ \\
\hline 610 & & 4347 (2337-6210) \\
\hline 611 & & $\begin{array}{c}503(82.3 \%) \text { female } \\
108(17.7 \%) \text { male }\end{array}$ \\
\hline 610 & & 145/610 (23.8\%) \\
\hline 610 & & $29 / 610(4.8 \%)$ \\
\hline 610 & & $61 / 610(10 \%)$ \\
\hline 610 & & $81 / 610(13.4 \%)$ \\
\hline 610 & & $91 / 610(14.9 \%)$ \\
\hline 610 & & $95 / 610(15.6 \%)$ \\
\hline 610 & & $168 / 610(27.5 \%)$ \\
\hline 611 & & 100/611 (16.4\%) \\
\hline 611 & & $88 / 611(14.4 \%)$ \\
\hline 611 & & 119/611 (19.5\%) \\
\hline 611 & & 18/611 (2.9\%) \\
\hline 611 & & $61 / 611(10.0 \%)$ \\
\hline 611 & & 102/611 (16.7\%) \\
\hline 611 & $2.1-2.6$ & $2.73(2.66-2.84)$ \\
\hline 611 & $2.1-2.6$ & $2.72(2.65-2.84)$ \\
\hline 611 & $2.1-2.6$ & $2.85(2.75-2.99)$ \\
\hline 611 & $1.6-7.5$ & $12.1(9.3-17.2)$ \\
\hline 413 & $>50$ & $31(22-48)$ \\
\hline 584 & $0.8-1.4$ & $0.90(0.77-1.01)$ \\
\hline 243 & $0.7-1.0$ & $0.84(0.73-0.91)$ \\
\hline 611 & $60-120$ & $71(63-87)$ \\
\hline 611 & $40-125$ & $94(78-114)$ \\
\hline 334 & & $3.6(2.0-5.3)$ \\
\hline 216 & $<7.5$ & $6.1(3.8-9.0)$ \\
\hline 220 & & $0.0158(0.0091-0.0213)$ \\
\hline
\end{tabular}

reported no symptoms consistent with PHPT. Fatigue (2.88 (2.81-3.01) vs $2.84 \mathrm{mM} \quad(2.73-2.98), \quad P=0.002)$ and weakness (2.94 (2.86-3.09) vs $2.84 \mathrm{mM}$ (2.75-2.98), $P=0.002)$ were associated with higher peak calcium but not PTH, adjusted calcium or calcium at presentation. Polydipsia was associated with higher adjusted calcium (2.77 (2.64-2.92) vs $2.72 \mathrm{mM}$ (2.65-2.82), $P=0.041)$, higher peak calcium (2.92 (2.81-3.06) vs 2.84 (2.75-2.96), $P<0.001)$ and higher PTH (14.2 (10.8-21.1) vs $12.0 \mathrm{pM}$ (9.2-16.5), $P=0.002)$. Similarly polyuria was associated with higher adjusted calcium (2.76 (2.67-2.91) vs 2.72 (2.65-2.83), $P=0.020$ ) and peak calcium (2.89 (2.80-3.06) vs $2.84 \mathrm{mM}(2.75-2.96), \quad P=0.005)$. Constipation or abdominal pain was associated with higher presenting calcium (2.77 (2.68-2.90) vs $2.73(2.66-2.83), P=0.016)$ but not peak calcium, adjusted calcium or PTH. Logistic regression analysis identified peak calcium (fatigue (OR 3.304, $P=0.028$ ), polydipsia (OR 7.512, $P=0.012)$ ) and PTH (polyuria $(1.014, P=0.045)$ ) as being independently associated with symptoms; full analysis and logistic

$\begin{array}{lr}\text { https://ec.bioscientifica.com } & \text { ○ } 2018 \text { The authors } \\ \text { https://doi.org/10.1530/EC-18-0195 } & \text { Published by Bioscientifica Ltd }\end{array}$

regression models are presented in Supplementary Table 1 (see section on Supplementary data given at the end of this article). Memory impairment, depression and bone/joint pain were not independently associated with biochemical indices of PHPT.

\section{Clinical features}

Previous bone fracture was present in $16.4 \%(n=100)$ at presentation with no significant clinical or biochemical (including PTH and calcium) associations observed (Supplementary Table 2A). A history of nephrolithiasis was present in $10.0 \%$ at initial presentation $(n=61)$ and was associated with higher adjusted calcium $(2.80$ (2.69-2.92) vs $2.72 \mathrm{mM}(2.64-2.82), P=0.002)$ and PTH (13.2 (10.3-22.0) vs 12.1 (9.3-16.7), $P=0.043)$, although in logistic regression analysis, younger age (OR 0.974, $P=0.006)$ and male gender (OR 1.972, $P=0.037$ ), but not biochemical indices, were associated with the presence of renal stones at presentation (Supplementary Table 2B).

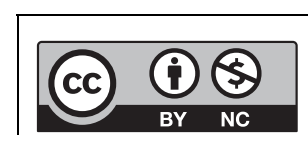

This work is licensed under a Creative Commons Attribution-NonCommercial 4.0 International License. 
Cardiovascular disease was present in $16.7 \%(n=102)$ at diagnosis of PHPT and hypertension in $43.5 \%(n=266)$, neither were independently associated with biochemical indices of PHPT (Supplementary Table 2C and D).

$19.5 \%(n=119)$ of patients were on a thiazide diuretic (specifically, bendroflumethazide) at diagnosis with no independently significant differences in biochemistry between thiazide-treated and non-thiazide-treated patients (Supplementary Table 2E). Standard practice was to discontinue thiazide diuretics in all patients with PHPT. At 1-year follow-up ( $n=215-$ no parathyroid surgery and 1 -year data available), there was no significant difference in the change in adjusted calcium: $-0.01(-0.11$ to 0.06$)$ in thiazide exposed vs $-0.04 \mathrm{mM}(-0.11$ to 0.04$)$ with no previous thiazide, $P=0.514$.

\section{Correlation analyses}

PTH was not correlated with age or BMI but was associated with adjusted calcium $(R 0.350, P<0.001)$, urinary CCCR $(R 0.145, P<0.05)$ and tumour volume $(R 0.494, P<0.001)$. A weaker association existed between tumour volume and adjusted calcium $(R 0.246, P<0.001)$. Vitamin D was only significantly inversely correlated with PTH $(R-0.270, P<0.001)$. The full correlation matrix is presented in Table 2 .

\section{Discriminatory value of CCCR}

CCCR was measured in 220 patients (36.0\%). Urine CCCR did not differ significantly between individuals with vitamin D sufficiency (0.017 (0.009-0.024), $n=45)$, insufficiency (0.016 (0.009-0.020), $n=72)$ and deficiency (0.015 (0.009-0.021), $n=50, P=0.612$ ) (Fig. 1). Similarly, urine CCCR did not significantly differ between spot urine samples (0.015 (0.010-0.023), $n=104)$ and 24-h urine collection samples (0.016 (0.010-0.023), $n=116$, $P=0.239)$. CCCR was $<0.01$ in $18 / 99(18.2 \%)$ surgically cured cases of PHPT and was $<0.02$ in $60 / 99$ (60.6\%).

\section{Neck imaging}

$64.8 \%(n=396)$ of patients received at least one modality of neck imaging. Independent predictors of neck imaging in this cohort included younger age (OR 0.912, $P<0.001$ ), higher adjusted calcium (OR 38.3, $P<0.001$ ), lower phosphate (OR 0.05, $P<0.001$ ), higher PTH (OR 1.03, $P=0.02)$ and presence of renal stones at presentation (OR 2.5, $P=0.046$ ). The presence of osteoporosis or previous fracture was not associated with the decision to request neck imaging (full results presented in Supplementary Table 3A).

Neck ultrasound was performed in $61.2 \%$ of patients $(n=374)$, of whom an adenoma was reported as being identified in $66.0 \% \quad(n=247)$. Failure to identify an adenoma on ultrasound was associated with older age (66 (60-77) vs 62 years $(51-71), P<0.001)$, lower peak calcium (2.86 (2.78-3.00) vs $2.91 \mathrm{mM}(2.82-3.05), P=0.014)$, lower PTH (12.0 (8.7-16.6) vs $14.0 \mathrm{pM}(10.0-19.7), P=0.006)$ and higher creatinine (72 (64-87) vs $69 \mu \mathrm{M}(61-81), P=0.022)$. Age (OR 0.963, $P<0.001$ ), PTH (OR 1.027, $P=0.043$ ) and creatinine (OR 0.989, $P=0.047$ ), were all independently predictive in logistic regression analysis (presented in full in Supplementary Table 3B). The relationship with age remained present when the analysis was limited to patients with subsequent surgical cure (OR 0.963, $P<0.001)$ and so does not represent misdiagnosis of PHPT. Tumour volume and weight (at subsequent surgery) were not associated with the failure of ultrasound to detect an adenoma (Supplementary Table 3B).

Where an adenoma was identified on ultrasound, the laterality was confirmed to be correct at surgery in $94.5 \%$ of cases $(172 / 182)$. The corresponding figure for sestamibi uptake was $66.1 \%(76 / 115)$; for the CT component of SPECT CT, this was $83.3 \%(35 / 42)$. The number of 4 DCT scans was substantially lower, but 7/10 adenomas reported were on the correct side at surgery. The full details of precise localisation (i.e. correct laterality and whether superior/inferior) are presented in Table 3 for patients who had parathyroid surgery. The proportion of imaging studies that identified an adenoma and how this related to non-curative surgery are presented in Table 4 .

\section{Delayed diagnosis and treatment}

The interval between an elevated calcium and measurement of PTH was less than 1 year in $72.5 \%$ of patients $(n=443)$ but was between 1 and 5 years in 15.5\% $(n=95)$ and greater than 5 years in $11.9 \%(n=73)$. Patients with a delayed diagnosis were younger (62 (51-60) vs 67 years (57-66), $P=0.004)$ and had lower initial adjusted calcium concentration (2.64 (2.59-2.72) vs 2.76 (2.59-2.72), $P<0.001$ ) but no difference in PTH (full results presented in Supplementary Table 4).

Where the diagnosis of PHPT was made within 1 year, $26.1 \%$ received surgery within 1 year, $18.9 \%$ between 1 and 5 years and $1.6 \%$ beyond 5 years (no surgery in $53.4 \%$ ). Where the diagnosis was made between 1 and 5 years, $31.2 \%$ received surgery within this timeframe and 


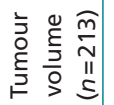

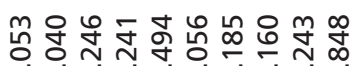
i 000 o

$\underset{\substack{\infty \\ 0 \\ 0}}{1}$

亏 芯命

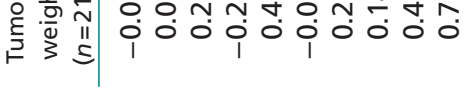

$\overline{8}$
v
v

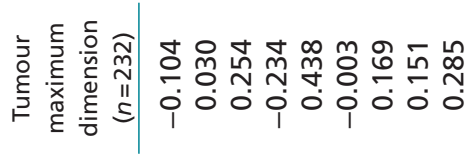

$\bar{\delta} \bar{\delta}$
$\dot{0} \dot{0}$
$\dot{0}$

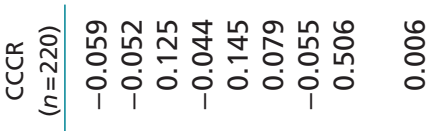

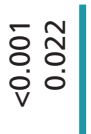

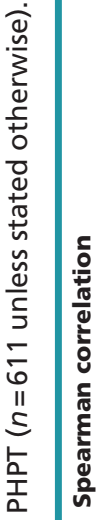

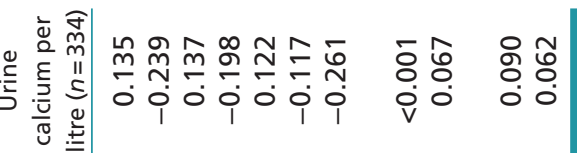

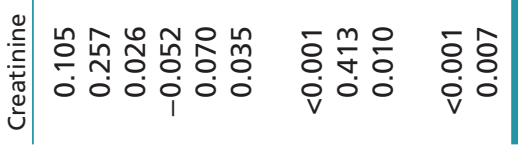

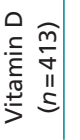

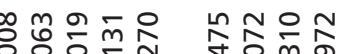

000.010

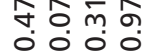

\section{I}

000 i

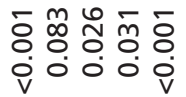

苋

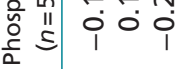

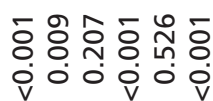

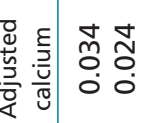

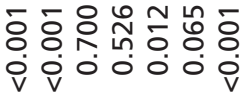

苂

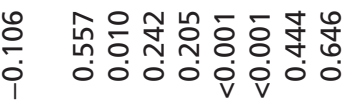

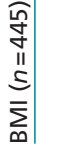

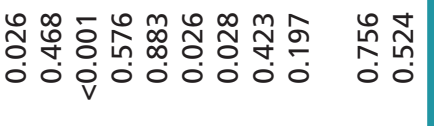

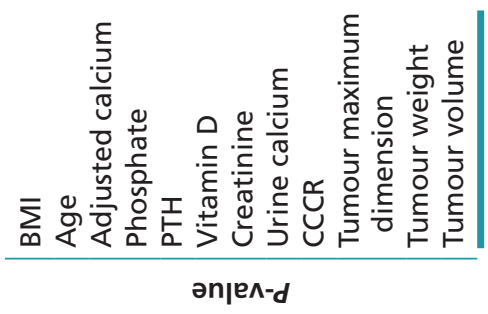

https://ec.bioscientifica.com

https://doi.org/10.1530/EC-18-0195

(C) 2018 The authors Published by Bioscientifica Ltd a further $7.5 \%$ received surgery beyond 5 years (no surgery in $61.3 \%$ ). Where the diagnosis was delayed beyond 5 years, 38.3\% received surgery (no surgery in 61.6\%).

\section{Features associated with having parathyroid surgery}

$44.8 \%$ of our patients $(n=274)$ had parathyroid surgery. Surgery was more likely in younger individuals (61 (50-70) vs 74 years $(66-81), P<0.001)$, those with higher adjusted calcium (2.78 (2.68-2.92) vs $2.70 \mathrm{mM}(2.64-2.77)$, higher PTH (14.3 (10.0-20.2) vs $11.4 \mathrm{pM}(8.8-14.3), P<0.001)$, where neck imaging identified an adenoma (OR 3.3, $P<0.001)$ and those with a history of nephrolithiasis (OR 1.80, $P<0.001)$. Osteoporosis was not associated with a higher likelihood of having had surgery (OR $0.89, P=0.273$ ), although after adjusting for age, there was a borderline association between osteoporosis and greater likelihood of surgery (OR 1.60, $P=0.057$ ). Logistic regression analysis confirmed identification of an adenoma on imaging (OR 10.7, $P<0.001$ ), younger age (OR 0.92, $P<0.001$ ), higher adjusted calcium (OR 15.3, $P<0.001)$ and history of nephrolithiasis (OR 3.1, $P=0.007$ ) as being independently associated with the likelihood of surgery (full analysis presented in Supplementary Table 5A, B and C).

\section{Predictors of non-curative surgery}

Surgery failed to cure PHPT in $8.4 \%$ of patients in our centre (23/274). There were no significant differences in preoperative clinical and biochemical parameters between those with curative and non-curative surgery (Supplementary Table 6). There was, however, an increased risk of non-curative surgery where more than one imaging modality was employed (OR 2.42, $P=0.035)$ and a trend towards higher likelihood of non-curative surgery in patients where US failed to identify an adenoma (OR 2.3, $P=0.052$ ). Of the 23 patients with non-curative surgery, 12 have had subsequent cure following reoperation. One further patient was subsequently diagnosed with FHH. Ultimately only $11 / 273$ (4.0\%) of patients with PHPT and surgical intervention had persistent PHPT.

\section{Location of tumour and pathology}

Where a precise location was specified following surgery ( $n=199)$, the right inferior position was the commonest adenoma site $(40.7 \%, n=81)$, followed by left inferior $(33.2 \%$, $n=66)$, left superior $(9.0 \%, n=18)$, right superior $(8.0 \%$, $n=16)$, multiple sites $(7.0 \%, n=14)$ and, finally, ectopic $(2.0 \%, n=4)$. The full tumour location data are presented

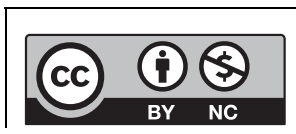

This work is licensed under a Creative Commons Attribution-NonCommercial 4.0 International License. 


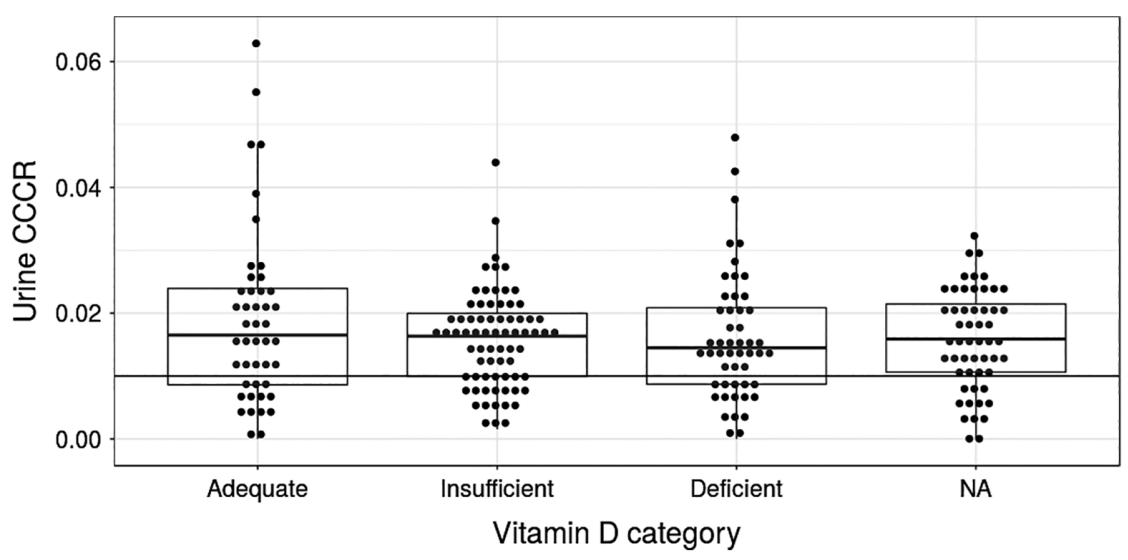

\section{Figure 1}

Urine CCCR presented across vitamin D categories. Horizontal line denotes 0.01 threshold $(P=0.612$ between the three vitamin $D$ categories). N/A not measured. in Supplementary Table 7. The commonest pathological diagnosis was adenoma $(79.8 \%, n=205)$, followed by hyperplasia $(12.8 \%, n=33)$, carcinoma $(4.3 \%, n=11)$ and atypical adenoma $(3.1 \%, n=8)$. Calculated tumour volume (R 0.494, $P<0.001$ ), maximum tumour dimension (R 0.438, $P<0.001)$ and tumour weight $(0.424, P<0.001)$ were all strongly correlated with PTH at diagnosis (Fig. 2).

\section{Discussion}

We have reported the largest detailed, consecutive series of patients presenting to a secondary care endocrine service for evaluation of PHPT. The results and conclusions drawn from this cohort are likely to be applicable to the majority of endocrine centres across the United Kingdom and beyond. Whilst susceptible to the typical criticisms of retrospective studies, the strength of this evaluation is its reflection of 'real world' clinical practice over the past decade.

\section{Symptoms}

Asymptomatic PHPT is often a misnomer as most patients with PHPT describe symptoms, which are at least consistent with hypercalcaemia. Previous studies of symptoms frequency have suggested over $90 \%$ of patients report at least one symptom $(11,12)$. These earlier studies were confined to patients awaiting parathyroid surgery and arguably represent the more severe end of the spectrum. The corresponding figure in our study was $71.1 \%$, although because we did not use a prospective data collection tool, this may reflect under-reporting. Whilst fatigue was relatively common in our patients (23.8\%), this was significantly lower than earlier reports (35-40\%) (11). In contrast, our reported prevalence of bone pain and polyuria were broadly consistent with previous estimates. Consistent with earlier reports, fatigue and polyuria appeared to be the symptoms most closely associated with biochemical indices of PHPT severity (12).

\section{Clinical features}

There is little doubt that PHPT is associated with an increased risk of nephrolithiasis. Recent estimates of the lifetime risk of nephrolithiasis in the general population are $3-5 \%$ in women and $10-15 \%$ in men (13). In our cohort, at presentation, $8.5 \%$ of women and $16.7 \%$ of men had a prior history of renal stones. Interestingly, whilst univariate analysis demonstrated significantly higher adjusted calcium in those with renal stones, logistic regression identified only younger age and male gender as independent associations. Indeed, in women

Table 3 Relationship between preoperative imaging and ultimate surgical outcome.

Correct location
Wrong location
No adenoma/uptake on imaging
No precise location reported by surgeon
No precise location reported by
radiologist
No adenoma at surgery
Multiple tumours at surgery
Total

\begin{tabular}{c} 
Ultrasound (\%) \\
\hline $116(46.4)$ \\
$18(7.2)$ \\
$56(22.4)$ \\
$48(19.2)$ \\
$0(0.0)$
\end{tabular}

$5(2.0)$
$7(2.8)$
250

https://ec.bioscientifica.com

https://doi.org/10.1530/EC-18-0195

\begin{tabular}{c}
${ }^{99 m}$ Tc-sestamibi uptake $(\%)$ \\
\hline $59(42.8)$ \\
$24(17.4)$ \\
$24(17.4)$ \\
$22(15.9)$ \\
$1(0.72)$ \\
$8(5.8)$ \\
$0(0.0)$ \\
138
\end{tabular}

\begin{tabular}{c}
\hline SPECT CT location (\%) \\
\hline $30(66.7)$ \\
$8(17.8)$ \\
$7(15.6)$ \\
$0(0.0)$ \\
$0(0.0)$ \\
$0(0.0)$ \\
$0(0.0)$ \\
45
\end{tabular}

\begin{tabular}{c} 
4DCT (\%) \\
\hline $7(53.8)$ \\
$0(0.0)$ \\
$3(23.1)$ \\
$0(0.0)$ \\
$0(0.0)$ \\
$3(23.1)$ \\
$0(0.0)$ \\
13
\end{tabular}


Table 4 Presence of enlarged parathyroid on imaging, whether surgery was performed and ultimate outcome of surgery by imaging modality and number of modalities employed.

\begin{tabular}{|c|c|c|c|c|c|c|c|}
\hline & 1 modality & 2 modalities & 3 modalities & 4 modalities & $\begin{array}{l}\text { Tumour on } \\
\text { scan? }\end{array}$ & Surgery done? & Surgery failed? \\
\hline Thyroid US & $168(44.9 \%)$ & $72(19.2 \%)$ & $121(32.4 \%)$ & $13(3.5 \%)$ & $247 / 374(66.0 \%)$ & $257 / 374(68.7 \%)$ & $20 / 257(7.8 \%)$ \\
\hline Sestamibi/sestamibi SPECT & $10(4.5 \%)$ & $76(34.5 \%)$ & $121(55.0 \%)$ & $13(5.9 \%)$ & $144 / 220(65.5 \%)$ & $137 / 220(62.3 \%)$ & $16 / 137(11.7 \%)$ \\
\hline SPECT CT & $0(0.0 \%)$ & $2(2.2 \%)$ & $78(83.9 \%)$ & $13(14.0 \%)$ & $64 / 93(68.8 \%)$ & $59 / 93(63.4 \%)$ & $6 / 59(10.2 \%)$ \\
\hline 4DCT & 0 & 0 & $18(60.0 \%)$ & $12(40.0 \%)$ & $15 / 30(50.0 \%)$ & $13 / 30(43.3 \%)$ & $4 / 13(30.8 \%)$ \\
\hline Tumour on any scan? & $166 / 181(91.7 \%)$ & $64 / 79(81.0 \%)$ & $99 / 121(81.8 \%)$ & $9 / 13(69.2 \%)$ & & & \\
\hline Surgery done? & $140 / 181(77.3 \%)$ & $51 / 79(64.6 \%)$ & $75 / 121(64.6 \%)$ & $6 / 13(46.2 \%)$ & & & \\
\hline Surgery failed? & $7 / 140(5.0 \%)$ & $7 / 51(13.7 \%)$ & $7 / 75(7.9 \%)$ & $2 / 6(33.3 \%)$ & & & \\
\hline
\end{tabular}

Where ${ }^{99 m}$ Tc-sestamibi SPECT CT was performed this is considered as two modalities (CT and scintigraphy). Planar ${ }^{99 m}$ Tc-sestamibi and ${ }^{99 m}$ Tc-sestamibi SPECT are reported as a single category.

first presenting over the age of 65 , the prevalence of renal stones is close to the upper end of prevalence estimates for the general population $(5.4 \%, 16 / 295)$. This suggests that aggressive case finding, with respect to nephrolithiasis, may be unnecessary in older women, although this would require validation in other populations. That the presence of cardiovascular disease and hypertension were not associated with biochemical indices of PHPT is not surprising given the high prevalence of these conditions and the multifactorial contributions to their development (14). Presence of previous fracture was also unrelated to presenting biochemistry again probably representing multifactorial contributions towards fracture risk.

\section{Thiazides}

As thiazide diuretics diminish urinary calcium excretion, received wisdom has been to discontinue this class of medication in patients diagnosed with PHPT, as this may reduce plasma calcium concentration. If thiazides do have a significant impact on plasma calcium we would have expected to see greater reduction in calcium at 1 year after their cessation in our non-surgical patients on thiazide diuretics at diagnosis (thiazides were discontinued in all patients in this cohort). However, no significant difference was observed lending further evidence to the contention (9) that the diagnosis of PHPT should not lead to automatic discontinuation of thiazides.

\section{CCCR}

Our demonstration that baseline urinary CCCR is less than 0.01 in $18.2 \%$ of patients with surgically confirmed PHPT calls into question the usefulness of this investigation in differentiating PHPT from FHH. This frequency is broadly consistent with previous reports $(15,16,17)$. Yet, the 4 th international workshop on the diagnosis of PHPT (8) suggests a greater than 95\% chance of FHH when CCCR is $<0.01$. This conclusion, which is commonplace in endocrine texts (18) is based on positive predictive value estimates performed in samples highly enriched with FHH cases, despite this being a substantially less common condition than PHPT (prevalence estimated at 1 in 78,000 in Scotland) (19).

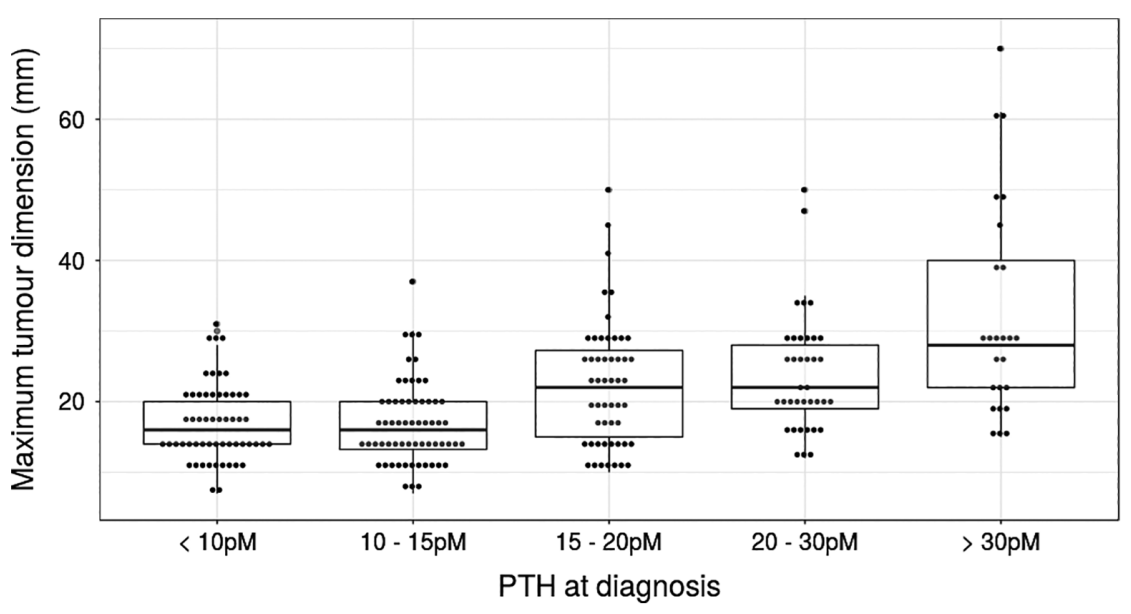

Figure 2

Maximum tumour dimension (median $\pm \mathrm{IQR}$ ) presented by PTH category at diagnosis (comparison across groups $P<0.001$ ).
○ 2018 The authors
Published by Bioscientifica Ltd

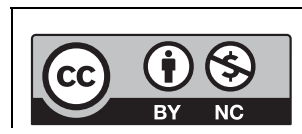

This work is licensed under a Creative Commons Attribution-NonCommercial 4.0 International License. 
PHPT remains by far the likeliest diagnosis in a hypercalcaemic patient with CCCR $<0.01$.

It is also frequently suggested that vitamin D status may complicate the interpretation of urinary CCCR results (8). However, we found no difference in urinary CCCR between vitamin D deficient, insufficient and replete individuals and no correlation existed between indices of urinary calcium excretion and plasma vitamin D. This lack of association is consistent with the observations in a small study of 35 PHPT patients (20) and also with the lack of effect upon urinary calcium excretion observed following vitamin D replacement in patients with PHPT (21).

We did not observe any difference in CCCR between 24-h samples and spot urine samples, although 24-h collections may be indicated for a full assessment of urinary calcium excretion in line with the current international workshop guidance (6).

\section{Imaging}

Failure of imaging to identify an adenoma was independently associated with a lower likelihood of proceeding to surgery and, in those who had surgery, failure to cure PHPT was more likely where more than one imaging modality was required. These facts strongly support the need for more effective second-line imaging modalities, as even 4DCT was associated with an appreciable failure rate. This study cannot be regarded as a 'head-to-head' comparison of imaging modalities as only more complex cases were referred for imaging beyond neck ultrasound. As a proportion of all ultrasound scans with subsequent surgery, only $46.4 \%$ identified the precise location (including cases where no adenoma was identified); however, $94.5 \%$ of scans where an adenoma was identified lateralised correctly. This compares to precise localisation in 78 (22), 74 (23), 61 (24) and 29\% (25) in previous studies. Differences in rates of adenoma identification between this cohort and others may reflect different population characteristics, as we identified older age and lower PTH to be independent predictors of failure to identify an adenoma. Evidence is mixed with respect to whether ${ }^{99 \mathrm{~m}} \mathrm{Tc}$-sestamibi is a superior initial investigation $(22,23,24,25)$ compared to ultrasound. Little difference appears to exist between planar ${ }^{99 \mathrm{~m}} \mathrm{Tc}$-sestamibi compared to ${ }^{99 \mathrm{~m}} \mathrm{Tc}$-sestamibi-SPECT (26), which supports our decision to consider these scans as a single entity in our analysis. 4 DCT is reported to lateralise correctly in $88 \%$ of cases and provide precise localisation in $70 \%$ (25). Although the number of 4DCT results in our cohort was low, it suggests this investigation does not perform this well in selected difficult cases where earlier imaging modalities have failed to identify abnormal parathyroid gland(s). Recent experience with 18-fluorocholine PET/CT offers some hope that this modality may offer improved performance in difficult cases where conventional imaging has failed to identify a source for PTH excess (27).

\section{Surgery}

We have confirmed the findings of earlier investigators that the majority of parathyroid adenomas (>70\%) occur in glands in the inferior position (28). Previous reports have offered conflicting evidence on the relationship between PTH at diagnosis and adenoma size $(29,30)$. However our series, which is the largest to address this question, shows an unequivocal relationship with respect to both calculated adenoma volume and weight. The prevalence of parathyroid carcinoma (4.3\%) was higher than is suggested elsewhere $(<1 \%$ of cases of PHPT) $(31)$, but this may be a consequence of referral bias i.e. 'milder' PHPT in older patients is less likely to be referred to secondary care and our population is therefore enriched with more severe disease. Otherwise the mix of pathological diagnoses is broadly in accord with the previous literature (1). Noncurative surgery was most strongly associated with difficulties in identifying disease on neck imaging, and this has been reported by other investigators previously (32). Earlier reports also identified older age, higher calcium and lower PTH as risk factors for failed surgery (33). Whilst our study was perhaps underpowered to reproduce these findings, trends were consistent with this larger series. Our centre has not utilised intraoperative PTH assessment, but this is a potentially attractive option, particularly in patients where risk factors for non-curative surgery are identified, to increase the likelihood of successful outcomes (34).

\section{Delayed diagnosis}

Over a quarter of patients in this cohort had a substantial delay between the first episode of hypercalcaemia and the diagnosis of PHPT, with $11.9 \%$ waiting over 5 years before PTH was measured. This is perhaps unsurprising given an earlier series where only $31 \%$ of hypercalcaemic patients had a PTH concentration assessed (4). Although it is difficult to quantify the potential harm inherent in delayed diagnosis, younger patients were more likely to https://ec.bioscientifica.com https://doi.org/10.1530/EC-18-0195 (c) 2018 The authors Published by Bioscientifica Ltd
This work is licensed under a Creative Commons Attribution-NonCommercial 4.0 International License. 
belong to this category and 38\% of those with greater than 5-year delay ultimately received surgery. In addition to exposure to symptoms and risk of classical PHPT complications, observational evidence associates elevated PTH with a greater risk of cardiovascular disease and mortality (35). Whilst we cannot infer causation from these observational data, it seems prudent to ensure timely diagnosis and expeditious referral for treatment in those where surgery is indicated. Our data suggest the need for improved systems and greater education in helping nonspecialists appropriately investigate patients with modest hypercalcaemia.

\section{Summary}

The insights gained from this cohort provide useful information on the most discriminant clinical features of PHPT and further reassurance that thiazide diuretic use is safe in PHPT. This series also suggests additional caution is required in the interpretation of CCCR and perhaps greater recourse to genetic testing, particularly as the cost of this technology plummets. With respect to imaging, we have demonstrated that neck ultrasound, when an enlarged parathyroid gland is positively identified, is highly accurate in lateralising disease. However, failure to identify an enlarged parathyroid, and the requirement for multiple imaging modalities, is a significant risk factor for non-curative surgery. Improved and novel imaging modalities are required to minimise the risk of unsuccessful surgery. Worryingly, it appears the delay in diagnosis of PHPT is unacceptably long in many patients, suggesting the need for better systems and better education for non-specialists to ensure prompt diagnosis and, where appropriate, treatment.

\section{Supplementary data}

This is linked to the online version of the paper at https://doi.org/10.1530/ EC-18-0195.

\section{Declaration of interest}

The authors declare that there is no conflict of interest that could be perceived as prejudicing the impartiality of the research reported.

\section{Funding}

This research did not receive any specific grant from any funding agency in the public, commercial or not-for-profit sector.

\section{Acknowledgement}

Thanks to Andrew Ditchfield and Andrew Brodie for their assistance in data collection for this project.

\section{References}

1 Bilezikian JP, Bandeira L, Khan A \& Cusano NE. Hyperparathyroidism. Lancet 2017391 168-178. (https://doi. org/10.1016/S0140-6736(17)31430-7)

2 Yeh MW, Ituarte PHG, Zhou HC, Nishimoto S, Liu IL, Harari A, Haigh PI \& Adams AL. Incidence and prevalence of primary hyperparathyroidism in a racially mixed population. Journal of Clinical Endocrinology and Metabolism 201398 1122-1129. (https:// doi.org/10.1210/jc.2012-4022)

3 Silverberg SJ, Clarke BL, Peacock M, Bandeira F, Boutroy S, Cusano NE, Dempster D, Lewiecki EM, Liu JM, Minisola S, et al. Current issues in the presentation of asymptomatic primary hyperparathyroidism: Proceedings of the Fourth International Workshop. Journal of Clinical Endocrinology and Metabolism 201499 3580-3594. (https://doi.org/10.1210/jc.2014-1415)

4 Balentine CJ, Xie R, Kirklin JK \& Chen H. Failure to diagnose hyperparathyroidism in 10,432 patients with hypercalcemia: opportunities for system-level intervention to increase surgical referrals and cure. Annals of Surgery 2017266 632-640. (https://doi. org/10.1097/SLA.0000000000002370)

5 Khan AA, Hanley DA, Rizzoli R, Bollerslev J, Young JE, Rejnmark L, Thakker R, D'Amour P, Paul T, Van Uum S, et al. Primary hyperparathyroidism: review and recommendations on evaluation, diagnosis, and management. A Canadian and International Consensus. Osteoporosis International 201728 1-19. (https://doi. org/10.1007/s00198-016-3716-2)

6 Bilezikian JP, Brandi ML, Eastell R, Silverberg SJ, Udelsman R, Marcocci C \& Potts JT. Guidelines for the management of asymptomatic primary hyperparathyroidism: summary statement from the Fourth International Workshop. Journal of Clinical Endocrinology and Metabolism 201499 3561-3569. (https://doi. org/10.1210/jc.2014-1413)

7 Udelsman R, Åkerström G, Biagini C, Duh QY, Miccoli P, Niederle B $\&$ Tonelli F. The surgical management of asymptomatic primary hyperparathyroidism: Proceedings of the Fourth International Workshop. Journal of Clinical Endocrinology and Metabolism 201499 3595-3606. (https://doi.org/10.1210/jc.2014-2000)

8 Eastell R, Brandi MLL, Costa AG, D'Amour P, Shoback DM \& Thakker RV. Diagnosis of asymptomatic primary hyperparathyroidism: Proceedings of the Fourth International Workshop. Journal of Clinical Endocrinology and Metabolism 201499 3570-3579. (https://doi.org/10.1210/jc.2014-1414)

9 Tsvetov G, Hirsch D, Shimon I, Benbassat C, Masrj-Iraqi H, Gorshtein A, Herzberg D, Shochat T, Shraga-Slutzky I \& DikerCohen T. Thiazide treatment in primary hyperparathyroidism - a new indication for an old medication? Journal of Clinical Endocrinology and Metabolism 2017102 1270-1276. (https://doi. org/10.1210/jc.2016-2481)

10 Scottish Government Offices. The Scottish index of multiple deprivation. (available at: http://www.gov.scot/Topics/Statistics/ SIMD). Accessed on 10 January 2018.

11 Udén P, Chan A, Duh QY, Siperstein A \& Clark OH. Primary hyperparathyroidism in younger and older patients: symptoms and outcome of surgery. World Journal of Surgery 199216 791-797.

12 Chan AK, Duh QY, Katz MH, Siperstein AE \& Clark OH. Clinical manifestations of primary hyperparathyroidism before and after parathyroidectomy. A case-control study. Annals of Surgery 1995222 402-414. (https://doi.org/10.1097/00000658-199509000-00017)

13 Rejnmark L, Vestergaard P \& Mosekilde L. Nephrolithiasis and renal calcifications in primary hyperparathyroidism. Journal of Clinical Endocrinology and Metabolism 201196 2377-2385. (https://doi. org/10.1210/jc.2011-0569)

14 Walker MD \& Silverberg SJ. Cardiovascular aspects of primary hyperparathyroidism. Journal of Endocrinological Investigation $2008 \mathbf{3 1}$ 925-931. (https://doi.org/10.1007/BF03346443)

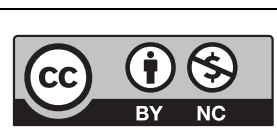

This work is licensed under a Creative Commons Attribution-NonCommercial 4.0 International License. 
15 Marx SJ. Letter to the Editor: Distinguishing typical primary hyperparathyroidism from familial hypocalciuric hypercalcemia by using an index of urinary calcium. Journal of Clinical Endocrinology and Metabolism 2015100 L29-L30. (https://doi.org/10.1210/jc.2014-4221)

16 Christensen SEE, Nissen PH, Vestergaard P, Heickendorff L, Brixen K \& Mosekilde L. Discriminative power of three indices of renal calcium excretion for the distinction between familial hypocalciuric hypercalcaemia and primary hyperparathyroidism: a follow-up study on methods. Clinical Endocrinology 200869 713-720. (https://doi. $\operatorname{org} / 10.1111 / \mathrm{j} .1365-2265.2008 .03259 . x)$

17 Jayasena CN, Mahmud M, Palazzo F, Donaldson M, Meeran K $\&$ Dhillo WS. Utility of the urine calcium-to-creatinine ratio to diagnose primary hyperparathyroidism in asymptomatic hypercalcaemic patients with vitamin D deficiency. Annals of Clinical Biochemistry 201148 126-129. (https://doi.org/10.1258/ acb.2010.010202)

18 Fuleihan GE \& Silverberg SJ. Primary hyperparathyroidism: diagnosis, differential diagnosis, and evaluation. (available at: https://www. uptodate.com/contents/primary-hyperparathyroidism-diagnosisdifferential-diagnosis-and-evaluation). Accessed on 10 January 2018.

19 Hinnie J, Bell E, McKillop E \& Gallacher S. The prevalence of familial hypocalciuric hypercalcemia. Calcified Tissue International 200168 216-218. (https://doi.org/10.1007/s002230001201)

20 Bussey AD \& Bruder JM. Urinary calcium excretion in primary hyperparathyroidism: relationship to 25-hydroxyvitamin D status. Endocrine Practices 200511 37-42. (https://doi.org/10.4158/ EP.11.1.37)

21 Grey A, Lucas J, Horne A, Gamble G, Davidson JS \& Reid IR. Vitamin $\mathrm{D}$ repletion in patients with primary hyperparathyroidism and coexistent vitamin D insufficiency. Journal of Clinical Endocrinology and Metabolism 200590 2122-2126. (https://doi.org/10.1210/ jc.2004-1772)

22 Tublin ME, Pryma DA, Yim JH, Ogilvie JB, Mountz JM, Bencherif B \& Carty SE. Localization of parathyroid adenomas by sonography and technetium Tc $99 \mathrm{~m}$ sestamibi single-photon emission computed tomography before minimally invasive parathyroidectomy: are both studies really needed? Journal of Ultrasound in Medicine 200928 183-190. (https://doi.org/10.7863/jum.2009.28.2.183)

23 Haber RS, Kim CK \& Inabnet WB. Ultrasonography for preoperative localization of enlarged parathyroid glands in primary hyperparathyroidism: comparison with $(99 \mathrm{~m})$ technetium sestamibi scintigraphy. Clinical Endocrinology 200257 241-249. (https://doi. org/10.1046/j.1365-2265.2002.01583.x)

24 Bhansali A, Masoodi SR, Bhadada S, Mittal BR, Behra A \& Singh P. Ultrasonography in detection of single and multiple abnormal parathyroid glands in primary hyperparathyroidism: comparison with radionuclide scintigraphy and surgery. Clinical Endocrinology 200665 340-345. (https://doi.org/10.1111/j.1365$2265.2006 .02601 \mathrm{x}$
25 Rodgers SE, Hunter GJ, Hamberg LM, Schellingerhout D, Doherty DB, Ayers GD, Shapiro SE, Edeiken BS, Truong MT, Evans DB, et al. Improved preoperative planning for directed parathyroidectomy with 4-dimensional computed tomography. Surgery 2006140 932-940. (https://doi.org/10.1016/j.surg.2006.07.028)

26 Nichols KJ, Tomas MB, Tronco GC, Rini JN, Kunjummen BD, Heller KS, Sznyter LA \& Palestro CJ. Preoperative parathyroid scintigraphic lesion localization: accuracy of various types of readings. Radiology 2008248 221-232. (https://doi.org/10.1148/ radiol.2481071066)

27 Thanseer N, Bhadada SKK, Sood A, Mittal BR, Behera A, Gorla AKR, Kalanthoorakahtu RR, Singh P, Dahiya D, Saikia UN, et al. Comparative effectiveness of ultrasonography, 99mTcSestamibi, and 18F-Fluorocholine PET/CT in detecting parathyroid adenomas in patients with primary hyperparathyroidism. Clinical Nuclear Medicine 201742 e491-e497. (https://doi.org/10.1097/ RLU.0000000000001845)

28 LoPinto M, Rubio GA, Khan ZF, Vaghaiwall TM, Farra JC \& Lew JI. Location of abnormal parathyroid glands: lessons from 810 parathyroidectomies. Journal of Surgical Research 2017207 22-26. (https://doi.org/10.1016/j.jss.2016.08.045)

29 Bindlish V, Freeman JL, Witterick IJ \& Asa SL. Correlation of biochemical parameters with single parathyroid adenoma weight and volume. Head and Neck 200224 1000-1003. (https://doi.org/10.1002/ hed.10165)

30 Randhawa PS, Mace AD, Nouraei SA \& Stearns MP. Primary hyperparathyroidism: do perioperative biochemical variables correlate with parathyroid adenoma weight or volume? Clinical Otolaryngology 200732 179-184. (https://doi.org/10.1111/j.13652273.2007.01447.x)

31 Givi B \& Shah JP. Parathyroid carcinoma. Clinical Oncology 201022 498-507. (https://doi.org/10.1016/j.clon.2010.04.007)

32 Yeh MW, Wiseman JE, Chu SD, Ituarte PH, Liu IL, Young KL, Kang SJ, Harari A \& Haigh PI. Population-level predictors of persistent hyperparathyroidism. Surgery 2011150 1113-1119. (https://doi. org/10.1016/j.surg.2011.09.025)

33 Cron DC, Kapeles SR, Andraska EA, Kwon ST, Kirk PS, McNeish BL, Lee CS \& Hughes DT. Predictors of operative failure in parathyroidectomy for primary hyperparathyroidism. American Journal of Surgery 2017214 509-514. (https://doi.org/10.1016/j. amjsurg.2017.01.012)

34 Bobanga ID \& McHenry CR. Is intraoperative parathyroid hormone monitoring necessary for primary hyperparathyroidism with concordant preoperative imaging? American Journal of Surgery 2017 213 484-488. (https://doi.org/10.1016/j.amjsurg.2016.11.035)

$35 \mathrm{Yu}$ N, Leese GP \& Donnan PT. What predicts adverse outcomes in untreated primary hyperparathyroidism? The parathyroid epidemiology and Audit Research Study (PEARS). Clinical Endocrinology 201379 27-34. (https://doi.org/10.1111/cen.12206)

Received in final form 14 August 2018

Accepted 22 August 2018

Accepted Preprint published online 23 August 2018 https://ec.bioscientifica.com https://doi.org/10.1530/EC-18-0195
() 2018 The authors Published by Bioscientifica Ltd

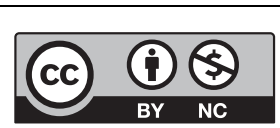

This work is licensed under a Creative Commons Attribution-NonCommercial 4.0 International License. 